Margareta Kampmann-Schwantes ${ }^{\mathrm{a}}$ Stefanie Breme-Breilmann ${ }^{b}$

a Oberkraemer/Schwante, Deutschland,

${ }^{b}$ Hamburg, Deutschland

\title{
Einmal Burnout und zurück
}

\section{Das Anliegen des Patienten}

Nach den guten Erfahrungen mit der Tagesklinik beabsichtigte der Patient nun, bei mir mit einer ambulanten Psychotherapie weiterzumachen. Er wollte wieder arbeitsfähig werden und seine sozialen Ängste abbauen. Er litt unter starken Stimmungsschwankungen, Schlafstörungen und häufigen Albträumen. Besonders belastet fühlte er sich durch einen Konflikt zwischen seiner Mutter und seiner Ehefrau. Es bereitete ihm Stress, mit seiner dreijährigen Tochter, die für ihn seinen Lebensmittelpunkt darstellt, auf den Spielplatz zu gehen, weil er oft das Gefühl hatte, sie vor den anderen Kindern schützen zu müssen. Körperlich fühlte er sich erschöpft und musste sich $\mathrm{zu}$ jeder Aktivität zwingen. In den letzten Jahren hatte er ca. $10 \mathrm{~kg} \mathrm{Ge-}$ wicht zugenommen. Last fallen wollen. Daher habe er um seine Kündigung gebeten und diese dann auch bekommen. Er habe «völlig neben sich gestanden», «nichts mehr auf die Reihe gebracht» und sich kaum noch aus dem Haus getraut. Schliesslich ging er für mehrere Wochen in eine psychosomatische Tagesklinik. Erst dort sei er «langsam wieder aufgetaucht». Es wurde ihm allmählich klar, dass er im Laufe mehrerer Jahre ein ausgeprägtes Burn-out-Syndrom entwickelt hatte.
Teil 1: Dr. Margareta Kampmann-Schwantes; Teil 2: Stefanie Breme-Breilmann.
- Die Labordiagnostik erbrachte einen Vitamin-D3- sowie einen Vitamin-B12-Mangel. Die anderen Parameter waren unauffällig. Eine Substitution wurde umgehend begonnen.

- Nach intensiver Beratung nahm der Patient eine Ernährungsumstellung basierend auf den Prinzipien einer Insulintrennkost vor. Gleichzeitig reduzierte er deutlich den Anteil an tierischem Protein und erhöhte den Anteil an frischem Obst und Gemüse. Er entwickelte wieder Freude am Kochen und war froh, auch seiner Frau und seinem Kind damit etwas Gutes zu tun.

- Bis der Patient auf seinem Fahrrad sass, war es ein langer Prozess. Früher viel auf seinem Rad unterwegs, rostete dieses jetzt vor sich hin. Vor allem Techniken aus der Verhaltenstherapie unterstützten ihn dabei, zunächst mit kleineren Spaziergängen anzufangen, dann sein Fahrrad wieder aus dem Keller zu holen, es zu reparieren und sich schliesslich zu trauen, wieder eine erste Runde zu fahren.

- Der Patient lernte als Entspannungsverfahren den «sicheren Ort» und führte ihn in eigener Regie überwiegend regelmässig durch.

Die Zusammenarbeit mit dem $\mathrm{Pa}$ tienten gestaltete sich von Anfang an sehr konstruktiv. In dem psychotherapeutischen Prozess wurden ihm biographische Zusammenhänge mit seiner jetzigen Situation bewusst und

\section{KARGER}

(C) 2015 S. Karger GmbH, Freiburg

Fax +497614520714 


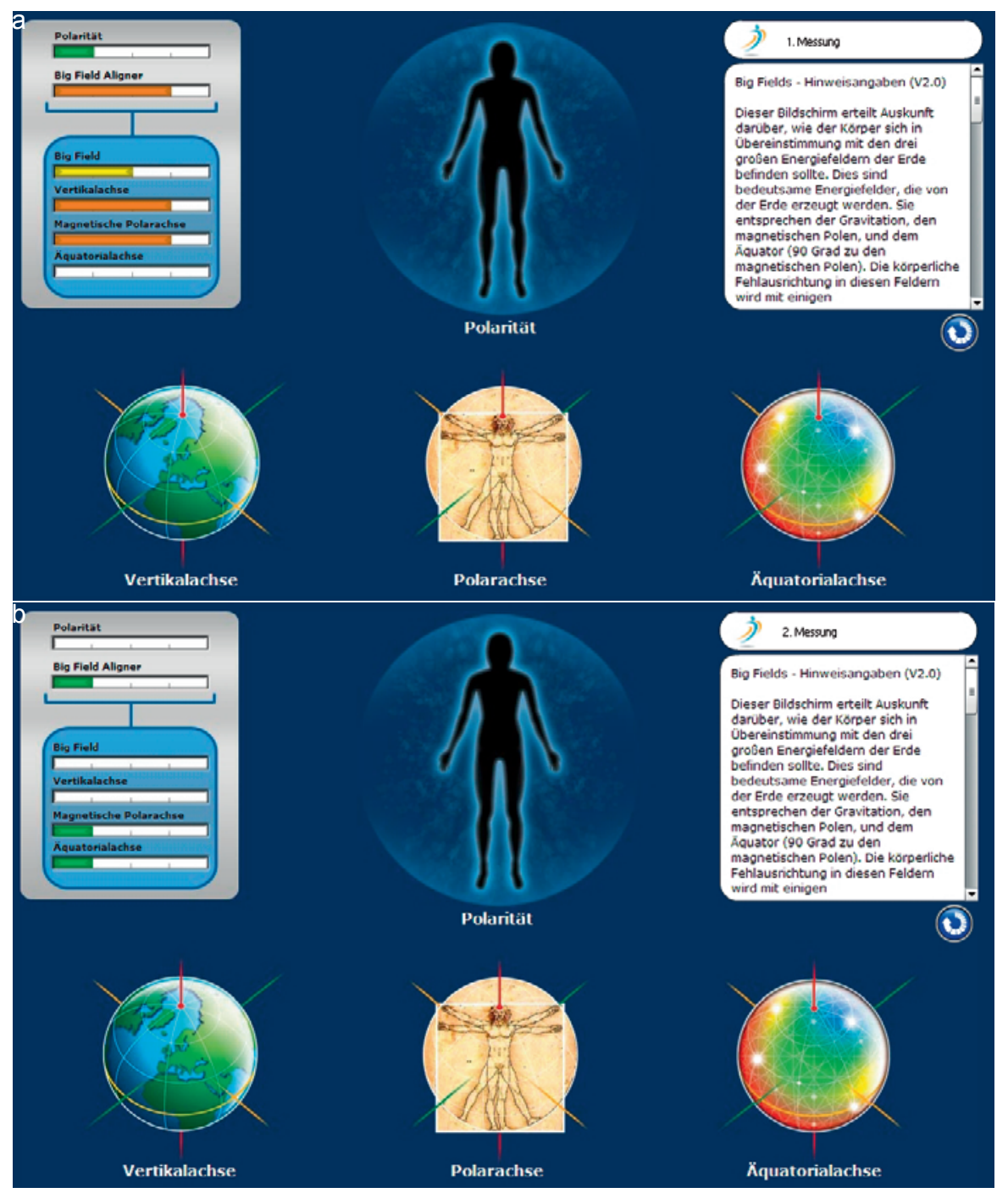

Abb. 1. Scanebene. a Der Scan zeigt deutliche Abweichungen des HBF von den grossen Energiefeldern der Erde. b 2 Monate später, nach der Behandlung mit Infoceuticals, zeigt sich ein weitgehend harmonisches $\mathrm{HBF}$

konnten gut bearbeitet werden. Er bekam wieder Zugang zu seinen eigenen Ressourcen und profitierte jetzt auch von seiner eigenen Ausbildung, unter anderem in systemischer Familientherapie.

Auch der körperliche Erholungsprozess setzte ein. Sein sich entwickelndes Selbstbewusstsein machte einen Sprung, nachdem er die ersten Kilos verloren und die ersten grösseren Radtouren hinter sich gebracht hatte. Er fing an, über seinen beruflichen Wiedereinstieg nachzudenken.

Der Patient konnte inzwischen mit seinen Ängsten zwar kompetent umgehen, aber es kostete ihn weiterhin viel Kraft, diese zu überwinden.
Nach ca. 6 Monaten Behandlung setzte ich eine neue Methode aus der Energie- und Informationsmedizin ein: NES Health. Ich wollte herausfinden, was diese Methode, die im Feinstofflichen ansetzt, an Erkenntnissen und weiteren Veränderungen bringen könnte.

Es handelt sich hierbei um ein quantenphysikalisches Verfahren, basierend auf der Vorstellung, Abweichungen in den Schwingungsmustern des quantenelektrodynamischen Körperfelds (Human Body Field (HBF)) festzustellen und diese mithilfe von Tropfen, sogenannten «Infoceuticals», wieder zu optimieren. Dem HBF wird dabei eine steuernde Funk- tion der gesamten energetischen und informativen Prozesse im Organismus zugeschrieben.

\section{Der neue Ansatz mit NES Health}

Wir führten also einen NES ProVision-Scan des Körperfelds durch, und meine Verblüffung war gross, wie gut das Ergebnis die Situation des $\mathrm{Pa}$ tienten und seine emotionalen Konflikte abbildete. Wir werteten die Ergebnisse in einem Gespräch ausführlich miteinander aus. Er nahm die «Infoceuticals» täglich und in aufsteigender Dosierung ein. Nach 2 Monaten wiederholten wir diese Prozedur.

Die Auswertung und die entsprechende Behandlung erfolgt bei dieser Methode auf mehreren Ebenen:

1) Zunächst werden die Abweichungen vom Körperfeld des Patienten von den grossen Energiefeldern der Erde gemessen. Das HBF meines Patienten wies dabei eine deutliche Abweichung auf (Abb. 1a).

2) Auf der psychischen Ebene zeigt das Hologramm des Cerebralen Cortex Kernkonflikte des Patienten auf (Abb. 2a). Seine Biographie lässt vermuten, dass diese bereits im vorsprachlichen Bereich (erstes bis drittes Lebensjahr) geprägt wurden. Bereits ab der achten Lebenswoche wurde er in einer Kindertagesstätte betreut und war schmerzhaften Bindungsverlusten sowie einer strengen preussischen Sauberkeitserziehung unterworfen. Unterordnung und Missachtung seiner Grenzen waren frühe und prägende Engramme. In der Therapie sind einzelne Erinnerungen aufgetaucht, schemenhafte Bilder, die vor allem mit dem Gefühl von Angst und Verlassenheit verbunden sind. Neben dem Gefühl «aushalten zu müssen», bis die Mutter «irgendwann» wiederkommt, erinnert er sich auch noch an andere Kinder, die von allen Seiten auf ihn zustürmen und ihm «Sachen wegnehmen». Hier liegt nicht nur der 


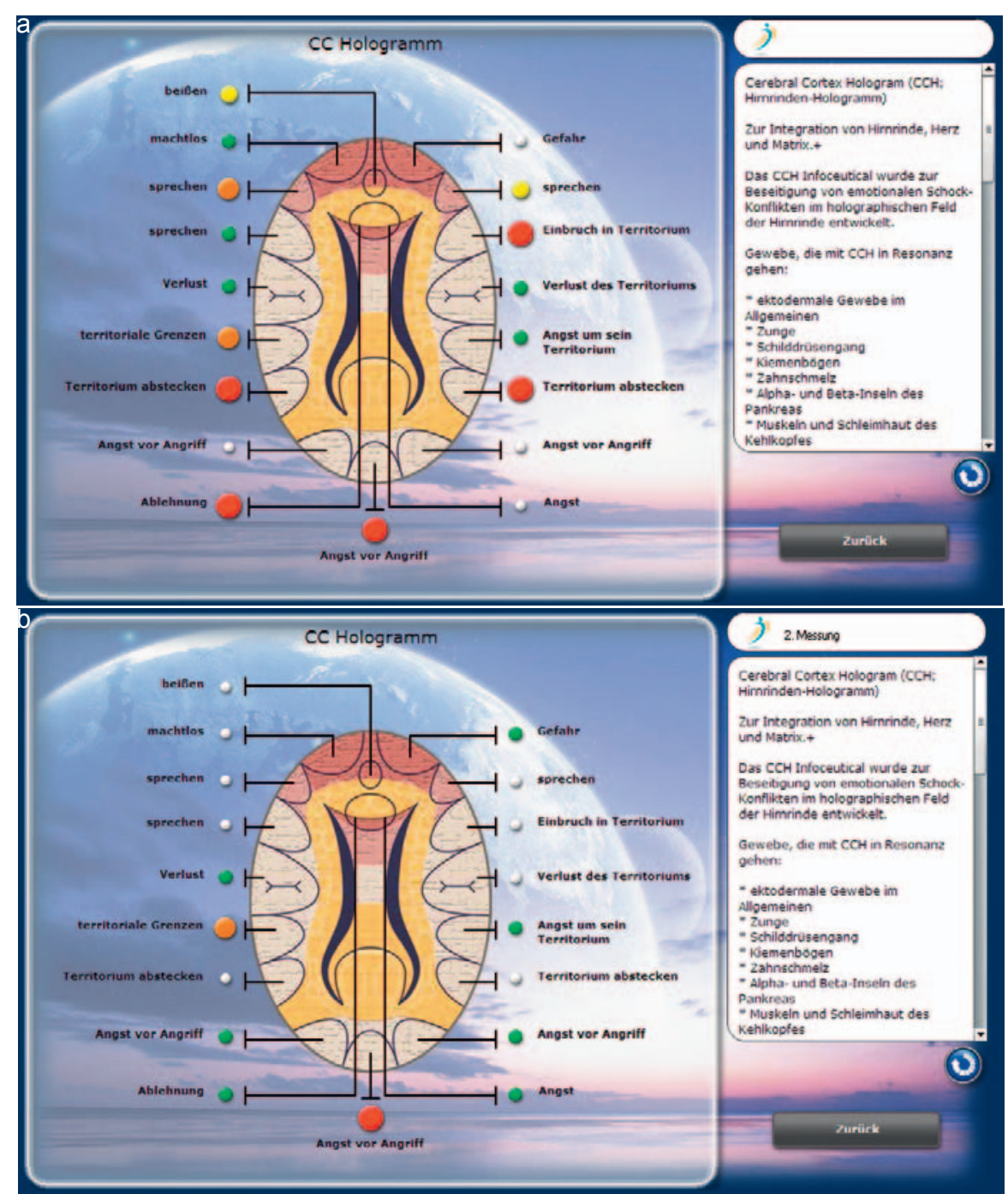

Abb. 2. Scanebene. a Das Hologramm des Cerebralen Cortex gibt Hinweise auf frühe traumatisch erlebte Grenzüberschreitungen in der Kita. b 2 Monate später, nach der Behandlung mit Infoceuticals, zeigt der Scan eine deutliche Reduktion dieses Kernkonflikts.

Grundstein für seine sozialen Ängste. Vor allem seine früh erlernte Fähigkeit, einfach zu warten, «auszuhalten» und eigene Bedürfnisse zu unterdrücken, statt sich zu wehren, war eine Prädilektionsstelle für das sich später entwickelnde Burnout (Abb. 2).

3) Die sogenannten «energetischen Treiber» sollen Auskunft über den energetischen Zustand der Felder von Organen bis auf die Zellebene geben. Hier zeigte die erste Messung eine ausgeprägte Störung im Feld der Informationsübertragung auf Zellebene. Dies könnte ein Korrelat zu der somatischen Ebene des Burnouts darstellen, mit der Er- der Erde zu harmonisieren (Big Field Aligner (BFA)), und ein zweites, um den Informationsaustausch auf Zellebene zu verbessern (Imprinter).

Ich hatte den Patienten auf die Möglichkeit von unangenehmen körperlichen Reaktionen (Übelkeit, Durchfall, Schwindel, Fieber und vieles andere mehr) hingewiesen. Diese sind als Ausdruck eines physischen und psychischen Reinigungsprozesses zu werten. Ähnliches kennt man aus der Homöopathie unter dem Begriff «Erstverschlimmerung». Wir hatten besprochen, wie er sich in diesem Falle verhalten sollte.

Bei unserem nächsten Termin 2 Wochen später erschien mir der Patient schon bei der Begrüssung verändert. Er sagte: «Das sind ja wirklich Zaubertropfen; meine Frau hat das auch gesagt». Er wirkte souveräner und war glücklich, weil die restlichen sozialen und klaustrophobischen Ängste, die ihn bis dahin noch belastet hatten, verschwunden waren. Unangenehme Erstreaktionen waren in seinem Fall ausgeblieben. Auch seine Träume hatten sich verändert. Er träumt auch jetzt noch weiterhin intensiv; Albträume (Abb. 4), die auch schon vor der Behandlung mit NES nachgelassen hatten, sind seither nicht mehr aufgetreten.

Nach 2 Monaten machten wir die nächste Messung und setzten die Therapie mit den Infoceuticals fort.

Inzwischen sind seit der ersten Messung 4 Monate vergangen. Der Patient ist wieder gesund und arbeitsfähig; Ängste sind bisher nicht mehr aufgetreten. Die Psychotherapie wird nach den nächsten zwei Sitzungen beendet sein.

Momentan sucht er mit Gelassenheit und Selbstbewusstsein eine neue Stelle, die seinen Fähigkeiten und Neigungen entsprechen soll. Vermutlich wird er ab Herbst dieses Jahres in einem Pilotprojekt eine Arbeit mit Jugendlichen beginnen und bereits in die Konzeptarbeit mit einbezogen sein. Auch auf beruflicher Ebene stellt dies für ihn einen Durchbruch dar. 


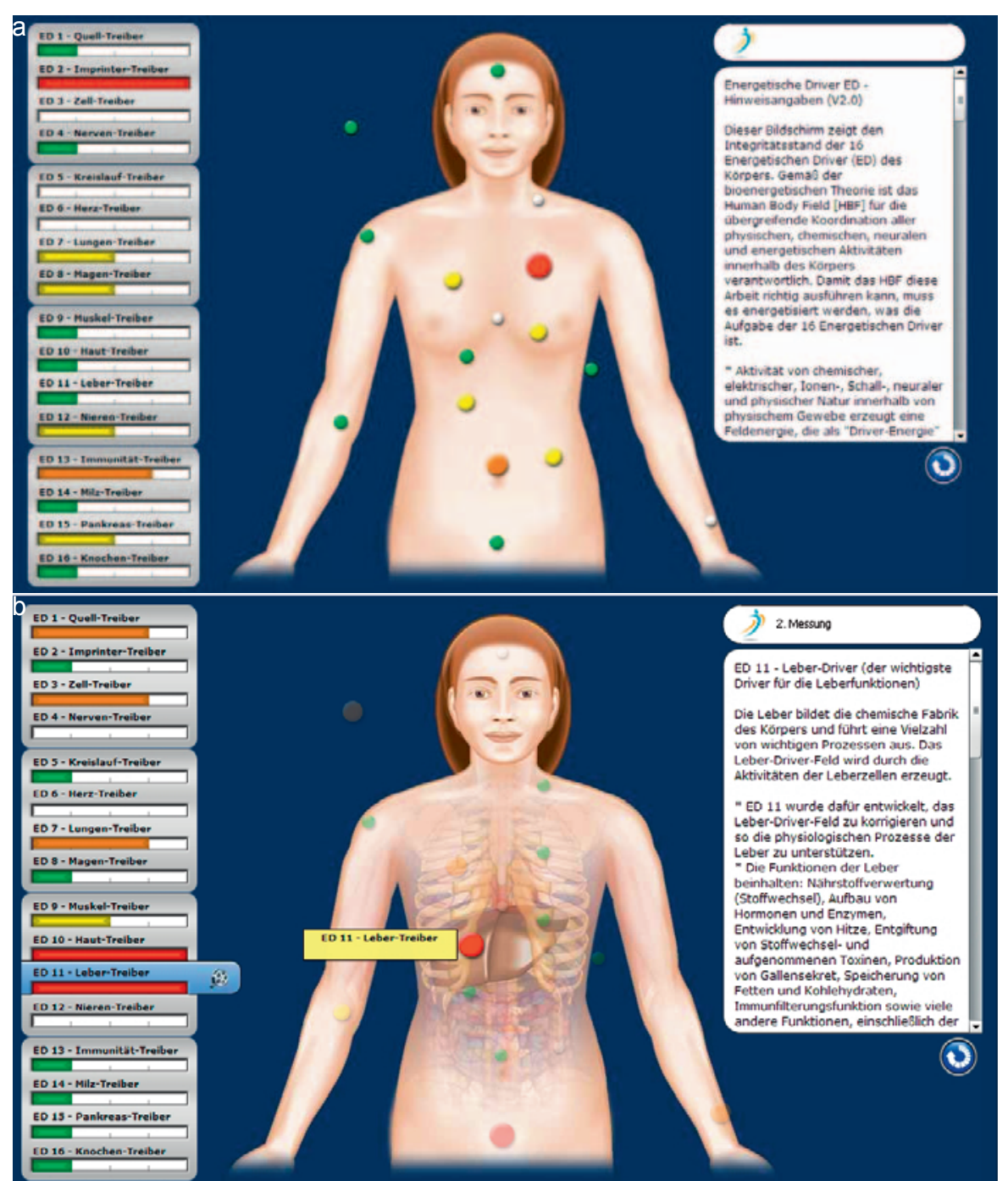

Abb. 3. Scanebene. a Auf energetischer Ebene zeigt sich eine Störung der Informationsübertragung auf Zellebene. b 2 Monate später, nach der Behandlung mit Infoceuticals, zeigt der Scan eine intensive Stoffwechselaktivität. Leber und Haut benötigen Unterstützung bei ihrer Entgiftungsfunktion.

Dieser Krankheits- und Heilungsverlauf ist für mich aus mehreren Gründen besonders interessant:

- Ein Patient, der sich in einem schweren Burnout befunden hatte, konnte sich unter einem multimodalen Therapieansatz innerhalb von 10 Monaten komplett regenerieren.

- Die erfolgreiche Bearbeitung seiner psychischen Konflikte, die dem Burn-out-Syndrom den Weg bereitet hatten, führte zu einem deutlichen Reifungsprozess und Kompetenzzuwachs des Patienten (posttraumatic growth).

\section{Teil 2: Die Herausforderung des Denkens - das menschliche Körperfeld}

Bei der von Frau Dr. KampmannSchwantes verwendeten Methode handelt es sich um ein neues Gesundheitssystem, das der - inzwischen verstorbene - australische Lehrstuhlinhaber für Akupunktur, Peter Fraser, entwickelt hat. Er forschte viele Jahre lang nach Heilungsmöglichkeiten für sein chronisches Erschöpfungssyndrom (chronic fatigue syndrome (CFS)) und fand heraus, dass der Schlüssel in einem komplexen (quanten-elektrodynamischen) Körperfeld (HBF) liegt, das die gesamten energetischen und informativen Prozesse im Organismus steuert.

Grenzwissenschaften belegen, dass es so etwas wie ein Informationsfeld geben muss. Dennoch gilt NES Health im schulmedizinischen Sinn als nicht wissenschaftlich abgesichert und teilt insofern den Status vieler komplementärmedizinischer Ansätze, bei denen aus der Sicht des Therapeuten nachweisbare subjektive und objektive Veränderungen entstehen. Infoceuticals beruhen auf einem ähnlichen Prinzip wie die Homöopathie, die auch mit einer Art Informationsträger für die Körperfeldinformation arbeitet und lange Jahre als nicht wissenschaftlich belegbar bekämpft wurde.

Fraser entwickelte in akribischer Detailarbeit ein Verfahren, in dem er dem HBF die fehlenden Informationen für die Selbstheilung zukommen lassen konnte (Infoceuticals und miHealth). So heilte er nicht nur sich selbst, sondern entwickelte auch eine bahnbrechende «Kartographierung» des HBF. In Harry Massey, einem Visionär und Unternehmer, fand Fraser den richtigen Partner und «Geburtshelfer» für das heutige NES Health System. Auch Massey litt damals unter CFS. Ihm gelang es, Frasers System in einem Softwareprogramm zu codieren und eine klinische Apparatur (NES ProVision) zu bauen, die das HBF einer Person scannen kann. 


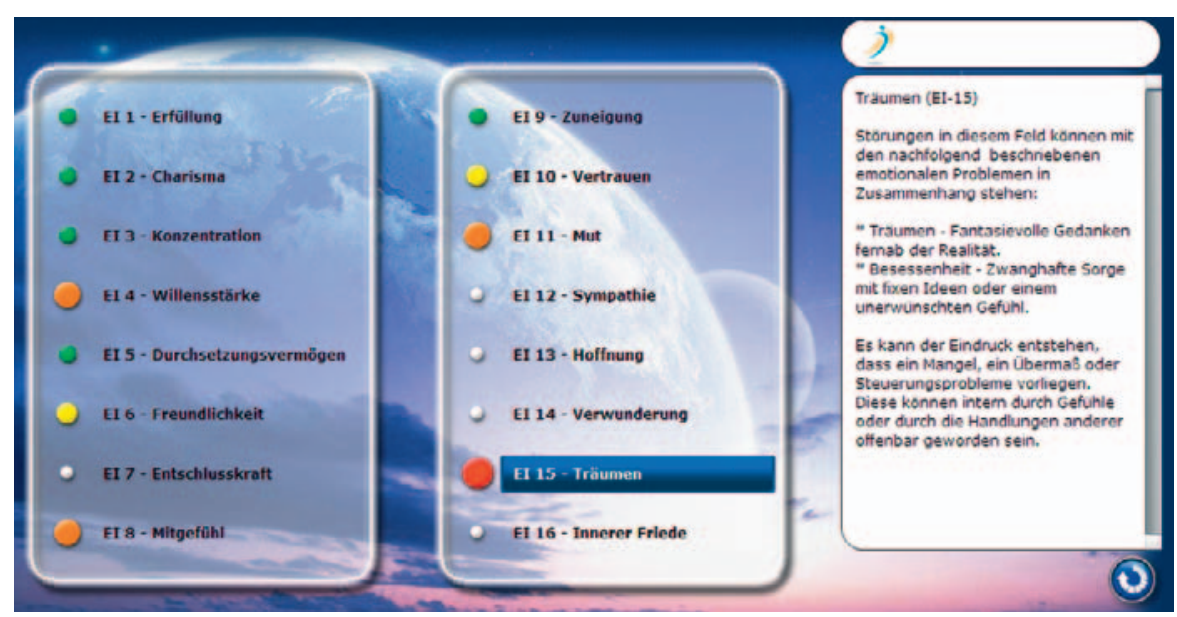

Abb. 4. Selbst die häufigen Albträume des Patienten finden bei der Messung des HBF ihren Niederschlag.

\section{Das Körperfeld mit NES ProVision} scannen

Beim Scannen geht das HBF mit all seinen, sich überlagernden Schwingungsmustern einzelner Organe, Organsysteme oder Meridiane mit den im Computer hinterlegten Schwingungsmustern in Resonanz. Ein Leber-Schwingungsmuster im Computer erkennt z.B. die gleiche Information beim Leber-Schwingungsmuster des Patienten. Dort, wo die Muster nicht in Resonanz gehen, «rauschen» sie aneinander vorbei. Als Ergebnis des Scanvorgangs werden alle Muster, die sich am wenigsten ähneln, als registrierte Abweichungen graphisch auf der Benutzerfläche der Software dargestellt.

Nach einem Scan von 20 s Dauer werden:

- über 400 Daten zum Gesundheitszustand des Patienten aus den Bereichen Information, Energie und Physiologie geliefert,

- insbesondere über die Felder der Organsysteme und Meridiane, über Ernährung und schädigende Umwelteinflüsse, über muskuloskelettale Störfelder sowie über psychische Ursachen, d.h. Emotionen einschliesslich Traumata und Schocks,

- eine Empfehlung nach dem Ursachenprinzip ausgesprochen,
- Hinweise für die entsprechende Behandlung mit den Infoceuticals gegeben,

- benutzerfreundliche klare Graphiken und Hilfstexte für den Behandler und den Patienten bereitgestellt.

So erhält der Therapeut schnell und ursachenbezogen Informationen, an welchen Stellen das Körperfeld offen für eine Korrektur ist und kann entsprechend therapieren.

\section{Optimierung des Körperfelds mit Infoceuticals}

Störungen im Schwingungsmuster des HBF können dann mithilfe von sogenannten «Infoceuticals» behandelt werden. Diese in Tropfenform codierten Schwingungsmuster sind die fehlenden Bausteine für eine optimale Kommunikation im HBF. Fraser und seinem Kollegen Massey ist es gelungen, über 70 verschiedene, in Zahlen codierte Feldinformationsmuster über ein Computerverfahren in einem 10000 Volt starken elektrostatischen Feld auf eine kolloidale Lösung von Mineralien aufzutragen. Diese Infoceuticals geben, einfach ausgedrückt, durch ihre eigenen Frequenzen Informationen (daher der Name) an das HBF und stimulieren es hiermit, wieder in die richtige Frequenz zu finden und so die Selbstheilungskräfte $\mathrm{zu}$ aktivieren.

\section{Die Herausforderung der}

Informationsmedizin - NES Health

Im obigen Fallbeispiel spricht der Patient von «Zaubertropfen». Er war bereit, sich auf NES Health ( $w w w$. neshealth.com; Kontakt: seminar@ sigridborchardt.de) einzulassen, ohne zu wissen, was genau passiert. Solange Symptome verschwinden, ist ein therapeutischer Ansatz willkommen. Doch nicht immer verschwinden die Symptome sofort und nicht selten geht der Selbstheilungsprozess des Körperfeldes über «Umwege». Die Rückenschmerzen bleiben zunächst, aber die Patientin wird z.B. mutiger in der Auseinandersetzung mit einer Kollegin. Bleiben wir in dem mechanistischen Denken von «Symptom - Diagnose Medikation - Symptombeseitigung», dann kann ein «Nicht-Sofort-Wirken» der Infoceuticals dazu führen, dass der Patient schnell aufgibt. Für Behandler und Patient besteht die Herausforderung in der neuen Sicht auf Gesundheit im Kontext eines sich selbst regulierenden nicht materiellen Informationsfelds und das Fürwahrhalten eines Modells von der Korrektur gestörter Felder, auf die wir einerseits Einfluss haben und die andererseits uns und unsere Emotionen prägen. Das ist schwer zu denken. Zudem können wir uns einfach nicht vorstellen, dass es unsichtbare, uns steuernde Muster im Raum gibt, von denen wir ein Teil sind. Nicht unser Verstand wiederholt nachprüfbare Prozesse, sondern das HBF reguliert sich selbst. Und genau hier, d.h. in diesem vertrauensvollen Loslassen unserer bisherigen Denkstrukturen, dem Loslassen einer Symptomorientierung und einer Bewertung von Veränderung, liegt unser Eigenanteil an der Heilung.

Der Medizin der Zukunft gelingt es also durchaus, bisher Entgegengesetztes zu vereinen: Geräte und Information, Materie und Geist, Körper und Bewusstsein, physiologische Therapien und geistiges Heilen. Und trotz materiell «empfangener» Diagnose und Medikation bleibt es der geistig- 
seelische Selbstheilungsprozess, den der Patient selbstverantwortlich annehmen kann oder auch nicht. In Bezug auf die Frage von Wissenschaft-

\section{Literatur}

1 Fraser P: NES - Das Nutri-Energetics System. CoMed 2/2007.

2 Fraser P, Massey H, Wilcox J: Decoding the Human Body Field. Rochester, VT, Healing Arts Press, 2008.

3 Massey H, Fraser P: The Unturned Stone (Der nicht umgedrehte Stein). Eine Revolution im präventiven Gesundheitswesen, ed 3. Selbstverlag, 2008. lichkeit treffen wir hier auf das bekannte Phänomen, dass die Erfahrung vorangeht und der Wissenschaft die Aufgabe der Nachprüfbarkeit über-

4 Meißner F: Quantenmedizin und das Human Body Field. CoMed 1/2008.

5 Meißner F: Quantenmedizin in der ganzheitsmedizinischen Praxis. CoMed 4/2008.

6 Viehweger R: Die Welt mit neuen Augen seh'n. Erkenne die Welt durch Global Scaling ${ }^{\circledR}$. Tübingen, RABS, 2010.

7 Viehweger R, Breme-Breilmann S: Die Macht des Feinstofflichen. CoMed 2/2014. lässt. Deshalb ist noch viel Forschung nötig, um die Evidenz zu erhärten.

8 Viehweger R: Über das menschliche Körperfeld. CoMed 11/2014.

9 Wolff M: Exploring the Physics of the Unknown Universe. Technotran, 1991.

10 Wolff M: Schrödinger's Universe und the Origin of the Natural Laws. Parker, CO, Outskirts, 2008. 